\title{
LAS 3R, MANO AMIGA EN LA ENSEÑANZA DE INGLÉS E INFORMÁTICA EN LA EDUCACIÓN RURAL
}

\section{THE 3R, FRIENDLY HAND IN TEACHING ENGLISH AND COMPUTING IN RURAL EDUCATION}

Estudiante Ana Maria Sierra Martínez ${ }^{\mathrm{a}}$, Estudiante Diana Marcela Guzmán Espitia ${ }^{\mathrm{b}}$, Lic. Leida Yamile Velásquez Camacho ${ }^{\mathrm{c}}$

a Corporación Universitaria Minuto de Dios “UNIMINUTO”, Semillero de investigación: Pedagogía Infantil UR3 UNIMINUTO "Recicla, reduce y reutiliza" Dirección Ave 15 Número 1 -22 Barrio la Fraguita, Zipaquirá, Colombia, asierramar2@uniminuto.edu.co

b Corporación Universitaria Minuto de Dios “UNIMINUTO”, Semillero de investigación: Pedagogía Infantil UR3 UNIMINUTO "Recicla, reduce y reutiliza" Dirección Ave 15 Número 1 -22 Barrio la Fraguita, Zipaquirá, Colombia, dguzmanespi@uniminuto.edu.co

c Corporación Universitaria Minuto de Dios “UNIMINUTO”, Semillero de investigación: Pedagogía Infantil UR3 UNIMINUTO "Recicla, reduce y reutiliza" Dirección Ave 15 Número 1 -22 Barrio la Fraguita, Zipaquirá, Colombia, lvelasque65uniminuto.edu.co

Recibido: 26-07-2015 / Aprobado: 12-10-2015

Resumen: El presente trabajo de investigación da a conocer cuáles son las causas que generan la ausencia de la enseñanza de las áreas inglés y tecnología e informática en los estudiantes de algunas Instituciones Educativas del sector rural de Pacho Cundinamarca, esta idea de investigación nace al reconocer la influencia de estos dos aspectos en la actualidad y el poco interés que aquí se ha dado. Al conocer la situación presentada con anterioridad se da paso a la creación y desarrollo de una propuesta innovadora para la enseñanza de estas dos áreas mediante la implementación de estrategias pedagógicas que permitan al estudiante interactuar directamente con el inglés y la informática creando así una experiencia de aprendizaje significativo. Pensando en hacer de este un trabajo investigativo de calidad se optó por el 
enfoque cuantitativo y la investigación acción participativa que fue aplicada a los estudiantes de grado segundo y tercero de la Institución Educativa Departamental Aquileo Parra sede rural La Cabrera; como resultados parciales se ha evidenciado que los estudiantes han tomado conciencia que estas dos áreas al igual que las demás tienen importancia en el desarrollo del estudiante y que no debe ser ni el contexto ni los recursos impedimento para aprenderlas.

Palabras clave: Enseñanza, Estrategia Pedagógica, Inglés, Propuesta Innovadora, Tecnología y Zona rural

Abstract: The present research project reveals the causes that generate the absence of the teaching of English and technology and informatics in the students of some Educational Institutions of the rural sector of Pacho Cundinamarca, this idea of investigation was born when recognizing the influence of These two aspects nowadays and the little interest that has been given here. To know the situation presented previously gives way to the creation and development of an innovative proposal for teaching these two areas through the implementation of pedagogical strategies that enable the student to interact directly with the English and computing, thus creating a significant learning experience. Thinking about making this a quality investigative work, we opted It has been chosen for the quantitative approach and the participatory action research that was applied to the second and third grade students of the I.E.D Aquileo Parra rural headquarter "La Cabrera"; As partial results it has been shown that the students have become aware that these two areas like the others are important in the student's development and should not be neither the context nor the resources impediment to learn them.

Keywords: Teaching, Pedagogical Strategy, English, Innovative Proposal, Technology, Rural zone. 


\section{INTRODUCCIÓN}

La presente investigación busca identificar las principales causas que impiden a los estudiantes de la zona rural recibir la misma "formación académica" que los estudiantes del sector urbano; la falta de equidad aquí es evidente, aun cuando que el Ministerio de Educación presenta una serie de lineamientos y estándares para determinadas áreas las cuales se consideran obligatorias, En algunos casos no se cumple a cabalidad en las instituciones educativas rurales, las cuales hacen sus modificaciones restando tiempo y dedicación a áreas que consideran de menor importancia para dar prioridad a las que ven como indispensables ignorando que la ausencia de la enseñanza de las mismas pueden ocasionar tropiezos a los estudiantes en un futuro.

Al pasar los años se van creando diversas metodologías para explicar un tema a determinado grupo, estas formas de enseñar se tienen en cuenta en algunas instituciones pero en otras no, las causas más evidentes frente al rechazo o poca aceptación de las metodologías son porque no lo creen necesario o conveniente, también porque trae más trabajo para el docente y este tiene establecido un mecanismo que años atrás le dio resultados, en fin son bastantes las situaciones que se han encontrado a lo largo de la investigación y estas se darán a conocer durante el desarrollo del documento.

Para nadie es un secreto que toda investigación nace de un interés sobre un tema, y aquí es un caso que sin saberlo afecta al futuro no solo de la comunidad educativa de la vereda La Cabrera, porque si bien es cierto el estudio se realizó en esta población pero es una problemática que se vive en diferentes rincones del país y del mundo en general; la idea aquí es esclarecer el por qué y plantear el cómo se puede dar tratamiento a la situación para que los estudiantes puedan recibir la enseñanza necesaria en las áreas de inglés, tecnología e informática de forma innovadora pensando siempre en llegar a todos de la mejor forma.

Para que el lector de este documento se sienta más contextualizado con proceso que se llevó a cabo le comentamos que después de identificar la problemática por medio de la observación directa al grupo, se dio paso a un diagnóstico de grupo el cual ayudo bastante porque aquí se conoció al estudiante, sus conocimientos previos y sus expectativas frente a las áreas a trabajar durante desarrollo del proyecto, a la vez se 
fue realizando un diagnóstico del contexto (Institución); después se establecieron unas metas a las cuales se pretendía llegar para eso se tuvo en cuenta las necesidades e intereses del estudiante, al docente director de grupo, el enfoque de la institución y la diversidad de recursos con los que se contaba, en fin se todo se desarrolló una serie de pasos (creación de hipótesis, recolección de datos, reunión de diversas fuentes de información sobre el tema, etc...) que arrojaron unos resultados y estos fueron tenidos en cuenta para crear y desarrollar una forma de enseñanza del inglés y la tecnología que busca ser lo suficientemente innovadora y convincente para que sea acogida en el contexto que se viene trabajando.

Como se mencionó anteriormente la meta o el objetivo que se busca es crear y desarrollar una propuesta innovadora para la enseñanza de las áreas inglés e informática a los estudiantes de primaria en la educación rural, teniendo como instrumentos de apoyo el uso de la tecnología y materiales reciclables. Para que eso se cumpla es indispensable plantear unos objetivos específicos los cuales acompañaran y facilitaran el recorrido de este trabajo; implementar estrategias pedagógicas, entre estas el uso de herramientas audiovisuales, de las Tics, el trabajo grupal y trabajos manuales para la enseñanza de las áreas a tratar. Para que estas estrategias se desarrollen es de vital importancia habilitar un espacio en la institución educativa donde los estudiantes puedan interactuar directamente con el inglés y la informática creando así un aprendizaje significativo por medio de la experiencia, al finalizar el proceso se busca presentar un entregable por eso se va a elaborar una cartilla didáctica la cual servirá como herramienta de enseñanza, teniendo en cuanta las necesidades encontradas en el contexto.

\section{METODOLOGÍA}

El tipo de investigación que se implemento tiene un enfoque cualitativo, cabe aclarar que es mediante la investigación cualitativa que se puede tener obtener más información sobre el tema, puesto que aquí no solo se basa en cantidades sino que también se reciben testimonios, experiencias, etc. Bien dicen Blasco y Pérez (2007:25), que "la investigación cualitativa estudia la realidad en su contexto natural y cómo sucede, sacando e interpretando fenómenos de acuerdo con las personas implicadas. Utiliza variedad de instrumentos para recoger información como la observación, diarios de campo, diario del 
investigador, entrevista, encuestas, historias de vida, en los que se describen las rutinas y las situaciones problemáticas, así como los significados en la vida de los participantes". Este proceso permite una hacer una construcción de un diagnostico real.

Ahora el tipo de investigación que se aplicó fue la "acción-participativa" expuesta esta por James McKernan (1999) como "un proceso de reflexión, por el cual en un área problema determinada, se desea mejorar la práctica o la comprensión personal, el profesional en ejercicio y este lleva a cabo un estudio en primer lugar, para definir con claridad el problema; en segundo lugar, para especificar un plan de acción; luego se emprende una evaluación para comprobar y establecer la efectividad de la acción tomada. Por último, los participantes reflexionan, explican los progresos y comunican estos resultados a la comunidad de investigadores de la acción. La investigación acción es un estudio científico auto reflexivo de los profesionales para mejorar la práctica".

Como grupo de investigación primero se realizó una observación detallada de una determinada población; para eso es indispensable contar con la activa participación de la comunidad educativa todo esto es para cumplir con un determinado fin que busca un beneficio para la población estudiantil.

Las personas que fueron participes de este estudio fueron los estudiantes de grado segundo y tercero de primaria pertenecientes a la Institución Educativa Departamental Aquileo Parra Sede rural La Cabrera. Municipio de Pacho Cundinamarca.

Dentro de las limitaciones que se presentaron podemos mencionar que cuando se iniciaron las clases de inglés, tecnología e informática algunos estudiantes no tenían el interés en estas áreas por el motivo que no se estaban enseñando con mucha frecuencia, pero los demás si tenían un interés por ellas; en el momento de las actividades lúdicas les parecía muy interesante, pero a la hora de lo teórico para ellos todo era muy aburridor y en ocasiones no hacían nada en esa parte de la actividad.

Había poco interés en algunos padres de familia; se llegó a conocer esto porque al dejar una como actividad para desarrollar en casa, los padres de familia no les exigían a sus hijos en realizar el trabajo y ellos tampoco brindaban una colaboración para hacer posible el desarrollo de la tarea, por 
este motivo los estudiantes se desanimaban mucho cumplir con sus trabajos.

Otro de los inconvenientes fue con una de las docentes que en el momento de iniciar la clase del área de inglés como estudiante de licenciatura en pedagogía infantil, se llevó una metodología diferente, en el momento la docente interrumpió la clase al pensar que no estaba haciendo lo correcto se le explico en qué consistía la actividad, pero no la dejo realizar, dando unas instrucciones en momento donde exigió cambiar lo planteado para cada una de las actividades.

Las herramientas que se utilizaron fueron: la observación, diarios de campo, diario del investigador, historias de vida, entrevista a un docente perteneciente a la Institución Educativa y a otro docente externa a la misma, también se realizó una encuesta a los estudiantes donde se pudo ver reflejado aspectos como el gusto por el inglés y la tecnología, su conocimiento sobre el tema, entre otros.

Dentro de las consultas sobre el tema cabe destacar autores que se tomaron como punto de referencia postulados como el de Noam Chomsky quien afirma que "La adquisición del lenguaje durante la infancia puede ocurrir gracias a la capacidad que tenemos los seres humanos de reconocer y asimilar la estructura básica del lenguaje, estructura que constituye la raíz esencial de cualquier idioma. Los niños poseen la habilidad innata para la comprensión de la gramática del lenguaje, habilidad que van desarrollando a través de sus experiencias y aprendizajes. Independientemente de su contexto familiar o cultural.

También estuvo presente Krashen quien considera que hay dos sistemas para adquirir una segunda lengua la adquisición y el aprendizaje; para él "la adquisición" es un proceso automático que se desarrolla en el nivel del subconsciente, debido a la necesidad de comunicación, no hay un esfuerzo consciente por parte del individuo ni un énfasis en el aspecto formal de la lengua, pero si en el acto comunicativo. Para que ocurra la adquisición es necesaria una gran interacción del individuo con la lengua. Mientras que el "aprendizaje" es un proceso consciente, debido al conocimiento formal de la lengua por adquirir. A través de este aprendizaje el individuo tiene la capacidad de explicar las reglas gramaticales en la lengua meta. La situación del aprendizaje contribuiría solo para dar un discurso poco fluido, ya que el individuo estaría más preocupado con la manera de que el mensaje es transmitido. 
En cuanto a la importancia de la enseñanza del área tecnología e informática el Lic. Joaquín Barragán Sánchez en su documento "Integración de tecnología en el proceso enseñanza-aprendizaje" dice que: "La integración de la tecnología al proceso enseñanza-aprendizaje no debe de ser una moda, una oferta promovida por los fabricantes de tecnología o bien como algo pasajero e intrascendente. El uso de tecnología, por sí misma, no resuelve los problemas de la educación, aunque su uso puede contribuir a evidenciarlos, buscar alternativas y propiciar nuevas situaciones de enseñanza- aprendizaje. Con la integración de tecnología se transformaría el proceso enseñanza aprendizaje. $\mathrm{La}$ tecnología debe ser una fuente de acceso al conocimiento y a las actividades de investigación y práctica en la comunidad educativa. La integración de tecnología la permitirá al alumno enfrentar exitosamente su vida personal, académica y profesional.

En el transcurso del desarrollo de la investigación siempre se buscó llegar a un punto donde los estudiantes vieran y crearan conciencia de la importancia de aprender inglés y tecnología y cómo estas áreas contribuían en la conservación del medio ambiente por eso cada una de las actividades se desarrollaron con materiales que en sus hogares ya no utilizaban y que para nosotros era nuestra materia prima.

\section{RESULTADOS}

Después de realizar un diagnóstico de grupo (Fig. 1 y 2) se determinó que a los estudiantes no les estaban brindando adecuadamente la enseñanza de dos áreas obligatorias como lo es lengua extranjera y tecnología e informática, esta situación debía ser solucionada loa antes posible.

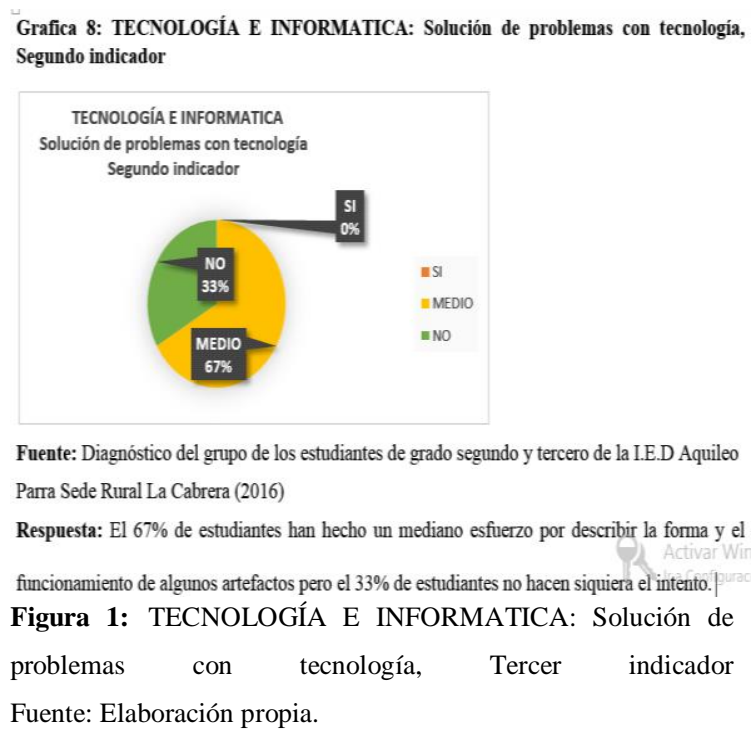

Fuente: Elaboración propia. 


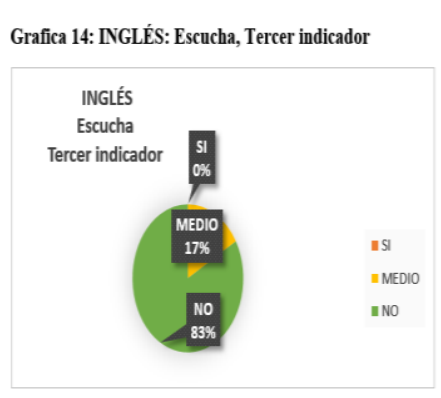

Fuente: Diagnóstico del grupo de los estudiantes de grado segundo y tercero de la I.E.D Aquileo Parra Sede Rural La Cabrera (2016)

Respuesta: En este punto hay falencias por que el $83 \%$ de los estudiantes NO Comprenden descripciones cortas, ni sencillas de objetos y lugares conocidos en inglés.

Figura 2: INGLÉS: Tercer indicador Fuente: Elaboración propia.

Al implementar la enseñanza de estas dos áreas utilizando recursos reciclables y tecnológicos se evidencio gran interés por parte de los estudiantes (Fig. 3); debido a este interés los contenidos temáticos han podido ser impartidos de una forma efectiva.

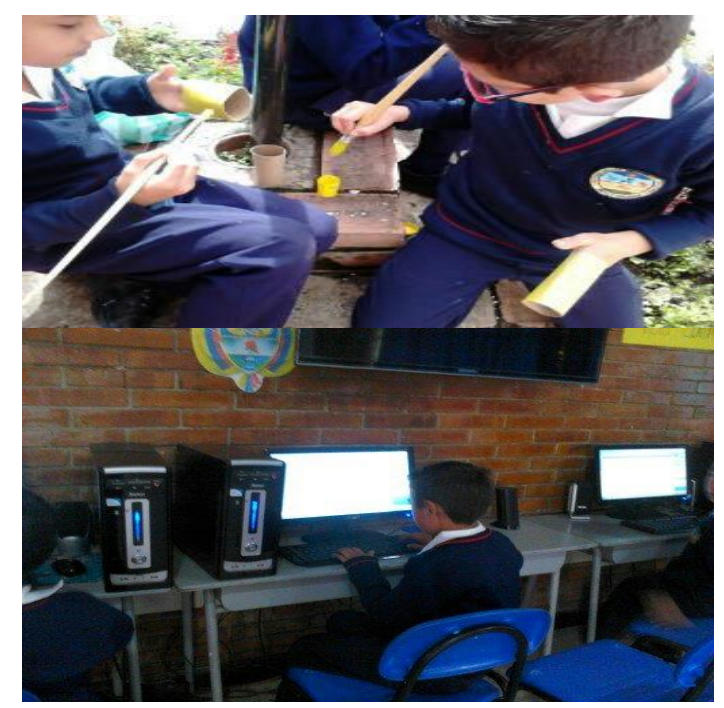

Figura3: enseñanza de inglés, tecnología e informática utilizando recursos reciclables y tecnológicos Fuente: Elaboración propia.
Los estudiantes han tomado conciencia que estas dos áreas al igual que las demás tienen una gran importancia en el desarrollo de cada persona y por eso no se deben pasar por alto en el calendario escolar.

El avance del conocimiento de los estudiantes en estas dos áreas ha sido notable; como las clases se han desarrollado de diferentes formas, ellos tienen presente las experiencias que estas clases les han dejado.

Por otro es fundamental mencionar que como resultado también se obtuvo la elaboración de dos cartillas una para la enseñanza de inglés y otra para tecnología e informática, cada una de ellas contiene una determinada cantidad de talleres didácticos para desarrollar, aplicando las metodologías que fueron empleadas durante el desarrollo del proyecto investigativo; lo que se quiere resaltar de estos entregables es que cada una de sus actividades fueron hechas pensando en el contexto rural y en las ventajas que este puede ofrecer a los estudiantes.

\section{CONCLUSIONES}

Se realizó diferentes actividades lúdico pedagógicas para obtener unos resultados coherentes con el contexto que se está tratando y así saber con certeza el conocimiento de cada uno estudiante, aquí 
se observó que no sabían mucho de las área de inglés e informática es por eso se concluyó que en la I.E.D Aquileo Parra Sede La Cabrera definitivamente no están fortaleciendo el desempeño de los estudiantes en estas dos áreas, una de las causas puede ser la falta de un docente capacitado para cada una de estas, otra causa evidente es que se ha restado importancia a estas dos materias en la zona rural.

Con base a estas áreas se realizaron actividades con materiales reciclables explicando cada uno de los temas básicos de inglés e informática, aquí se realizaban las tareas con materiales reciclables donde los estudiantes así mismo tenían mejores conocimientos a la hora de su realización y así mismo entendían cada uno de los temas expuestos.

Con los materiales que se iban realizando se adecuo un lugar para que cada estudiante interactuara con cada uno de los materiales lo analizara y a la vez aprendiera el inglés como se pronuncia como se escribe por otro lado el funcionamiento del computador; es muy importante tener en cuenta que siempre debemos adecuar el aula para que los estudiantes se sientan a gusto y se interesen por cada una de estas áreas porque es una buena motivación para ellos en su proceso de aprendizaje.

\section{FINANCIACIÓN}

En el desarrollo de este proyecto se logró concientizar a los estudiantes, padres de familia y docentes de la Institución Educativa Departamental Aquileo Parra sede rural La Cabrera sobre la influencia de la enseñanza de las áreas inglés, tecnología e informática hoy en día.

La participación activa de los niños y niñas de esta institución ha facilitado el camino para dar a conocer los temas elementales de estas dos áreas, el hecho de compartir sus experiencias en clase con su familia (y a la vez el apoyo que estas les han empezado a brindar) ha demostrado que se está avanzando en el tema.

La investigación se desarrolló de la mano del programa de licenciatura en pedagogía infantil perteneciente a corporación universitaria minuto de Dios UNIMINUTO, quienes apoyan los procesos por medio de los semilleros de investigación.

\section{BIBLIOGRAFÍA}

Adquisición de una segunda lengua. (Texto en línea) Teoría de Krashen. En línea. 
Disponible

en:

https://sites.google.com/site/adquisicionde unasegundalengua/teorias

Barragán Sánchez, Joaquín. (Documento PDF) Integración de tecnología en el proceso enseñanza-aprendizaje. En línea. Disponible en: http://bibliotecadigital.conevyt.org.mx/con curso/tematica_e/0132.pdf

Cerda Gutiérrez, Hugo. Cómo elaborar proyectos: Diseño, ejecución y evaluación de proyectos sociales y educativos. Bogotá: Cooperativa Editorial Magisterio, 2003 Pág. 29-44

Cerda Gutiérrez, Hugo. El proyecto de aula: el aula como sistema de investigación y construcción de conocimientos Tercera edición. Editorial Magisterio. Bogotá. Año: 2011 Pág. 188

Cumilaf, David Quidel; Rojas, Juan del Valle; López, Lissette Arévalo; Chihuaicura, Catherine Nancucheo; Neira, Ramón Ortiz. La enseñanza del idioma inglés a temprana edad: su impacto en el aprendizaje de los estudiantes de escuelas públicas, (2014). Revista de Comunicación Vivat Academia., No129 pp 34-56. Recuperado

de: http://search.proquest.com/openview/dabd 8b243fa66459291269dade291238/1?pqori gsite $=$ gscholar

Equipo de trabajo de la Oficina de Planeación Universidad Nacional de Colombia Sede Medellín, Guía Para la formulación de proyectos Considerando la metodología Del banco de proyectos de la Universidad nacional de Colombia (bpun), Noviembre 2007 Pág. 44 . En línea: http://www.bogota.unal.edu.co/planeacion/ download/herramientasmetodologia/Guia_ Formulacion_Proyectos.pdf

McKernan, James. Investigación -acciçon y curriculum. Ediciones Morata., S.L. (1999). En línea. Disponible en: http://www.terras.edu.ar/aula/cursos/10/bib lio/10MCKERNAN-James-Cap-1-Unfundamento-para-la-investigacionaccion.pdf 